\title{
PHOTOMETRIC VARIABILITY AT THE L/T DWARF BOUNDARY
}

\author{
Melissa L. ENOCH \\ Department of Astronomy, MS 105-24, California Institute of Technology, Pasadena, CA 91125; \\ menoch@astro.caltech.edu \\ Michael E. BRown \\ Division of Geological and Planetary Sciences, MS 150-21, California Institute of Technology, Pasadena, CA 91125; \\ mbrown@caltech.edu \\ AND \\ ADAM J. BURGASSER ${ }^{1}$ \\ Division of Astronomy and Astrophysics, UCLA, Los Angeles, CA 90095-1562; \\ adam@astro.ucla.edu \\ Received 2003 January 29; accepted 2003 April 29
}

\begin{abstract}
We have monitored the photometric variability of nine field $\mathrm{L}$ and $\mathrm{T}$ brown dwarfs for 10 nights during the course of 1 month. Observations were obtained in the $K_{s}$ band with the Palomar 60 inch $(1.5 \mathrm{~m})$ telescope Near-Infrared Camera. Results of statistical analyses indicate that at least three of the nine targets show significant evidence for variability, and three more are possibly variable. Fractional deviations from the median flux vary from $5 \%$ to $25 \%$. Two of the variable targets, 2MASS $0030-14$ (L7) and SDSS $0151+12$ (T1), have marginally significant peaks in their periodograms. The phased light curves show evidence for periodic behavior on timescales of 1.5 and $3.0 \mathrm{hr}$, respectively. No significant correlations between variability amplitude and spectral type or $J-K_{s}$ color are found. While it is clear that variability exists in objects near the $\mathrm{L} / \mathrm{T}$ dwarf boundary, we find no evidence that variability near the $\mathrm{L} / \mathrm{T}$ boundary is more likely than it is for early L dwarfs.
\end{abstract}

Key words: stars: individual (2MASSW J0030300-145033, SDSSp J015141.69+124429.6) — stars: low-mass, brown dwarfs — stars: variables: other

\section{INTRODUCTION}

In the last several years, two new low-mass stellar and brown dwarf spectral classes, L and T dwarfs, have been established (see, e.g., Martín et al. 1997, 1999; Kirkpatrick et al. 1999; Geballe et al. 2002; Burgasser et al. 2002c). Now that a method exists by which to classify these objects, efforts have turned to understanding their structure, origin, and evolution. One means of probing the atmospheric structure of $\mathrm{L}$ and $\mathrm{T}$ dwarfs has focused on the presence, or lack thereof, of photometric variability, which may be tied to magnetic activity or cloud properties (Ackerman \& Marley 2001).

Grain formation is likely an important process in the atmospheres of cool stellar and substellar objects, providing a significant source of opacity (e.g., Lunine et al. 1989). Iron and magnesium silicates such as enstatite should condense out and form droplets below about $2200 \mathrm{~K}$, leading to the formation of clouds (Burrows \& Sharp 1999). Current atmospheric calculations (e.g., Ackerman \& Marley 2001; Allard et al. 2001) find that early L dwarf spectra are best matched by a model atmosphere with thin silicate and iron clouds, late L dwarfs by an atmosphere with thick clouds, and $\mathrm{T}$ dwarfs by a clear atmosphere in which clouds presumably have fallen below the region from which flux emerges. The change in $J-K_{s}$ colors from progressively redder for later L types (Kirkpatrick et al. 1999, 2000; Leggett et al. 2001, 2002) to relatively blue for early $\mathrm{T}$ types (Leggett et al. 1999, 2002; Burgasser et al. 2002c) also suggests a

${ }^{1}$ Hubble Fellow. substantial atmospheric change across the L/T boundary. By assigning effective temperatures to each spectral subtype, Kirkpatrick et al. (2000) predict that the transition from $\mathrm{L}$ to $\mathrm{T}$ takes place over a very small range in effective temperature $(\leq 350 \mathrm{~K})$.

Burgasser et al. (2002b) have attempted to describe the transition in cloud cover that appears to occur at the $\mathrm{L} / \mathrm{T}$ transition by appropriately interpolating between clear and cloudy models (Ackerman \& Marley 2001). The authors find that such partly cloudy atmospheres can reproduce the colors and magnitudes of late $\mathrm{L}$ and early $\mathrm{T}$ dwarfs and suggest that substantial variability around $\lambda=1 \mu \mathrm{m}$ might be observed in this region due to the breakup of cloud layers, the appearance of long-lived cloud holes, or a combination of these effects.

It is unclear on what timescale such variability would occur, or how large a fractional variability amplitude to expect. A single large, long-lived feature or cloud hole might cause variability with a stable period, rapidly changing features would cause variability with no discernible period, and uniformly distributed features would not produce a variability signal at all (Bailer-Jones \& Mundt 2001; Gelino et al. 2002). As noted by Cooper et al. (2003), theoretical modeling of such a transition would require threedimensional cloud models, including convection, which do not yet exist for objects beyond the solar system.

Previous work has found several $\mathrm{M}$ and $\mathrm{L}$ stars and brown dwarfs to be photometrically variable in the optical (Tinney \& Tolley 1999; Bailer-Jones \& Mundt 1999, 2001; Martín, Zapatero Osorio, \& Lehto 2001; Clarke, Tinney, \& Covey 2002; Clarke, Oppenheimer, \& Tinney 2002; Gelino et al. 2002), but no dwarfs later than L5 were included in 
these samples. Nakajima et al. (2000) report near-IR spectroscopic variability in the T6 dwarf SDSS $1624+00$, while Kirkpatrick et al. (2000) find spectroscopic variability in the L8 dwarf G1 584C.

The observed variability could be a consequence of cloud structure, magnetic activity or spots, or some unknown phenomenon. Most variability studies, however, favor the cloud interpretation (Martín et al. 2001; Tinney \& Tolley 1999; Gelino et al. 2002). Gelino et al. (2002) argue that magnetic spots are probably not responsible for photometric variability in L dwarfs due to the low magnetic Reynolds number in their cool atmospheres. Given that detections of $\mathrm{H} \alpha$, one indicator of magnetic activity, drops dramatically in later $\mathrm{L}$ and $\mathrm{T}$ dwarfs (Kirkpatrick et al. 2000; Gizis et al. 2000) (there are, however, a few notable exceptions-Burgasser et al. 2002a), it seems unlikely that magnetic spots could cause variability in very late $\mathrm{L}$ or T dwarfs.

In an effort to better understand variability around the $\mathrm{L} / \mathrm{T}$ boundary, we have undertaken a program to photometrically monitor one peculiar early $\mathrm{L}$, four late $\mathrm{L}$, and four early $\mathrm{T}$ field dwarfs. In $\S 2$, we describe the observations and reduction of data. We present light curves for all nine targets and the results of variability analyses in $\S 3$. In $\S 4$, we briefly discuss our results in the context of ideas about cloud breakup at the L/T boundary, and $\S 5$ gives a short summary.

\section{OBSERVATIONS AND DATA REDUCTION}

Targets were observed during 2001 September 30October 6 and October 25-28 (UT), providing a total observational baseline of 29 days for each target. The first observation period, September 30-October 6, will hereafter be referred to as set 1, October $25-28$ as set 2 , and the complete duration of observations as the combined set. Observations were made with the $K_{s}(\lambda=2.17 \mu \mathrm{m})$ filter of the Near-Infrared Camera at the Palomar Observatory 60 inch Telescope. The instrument is a Cassegrain $256 \times 256$ pixel camera with a pixel scale of 0".619 pixel $^{-1}$ and total field of view of $6.98 \mathrm{arcmin}^{2}$. Bright late L and early T dwarf targets were chosen from the Two Micron All Sky Survey (2MASS; Skrutskie et al. 1997), the Sloan Digital Sky Survey (SDSS; York et al. 2000), and the Deep Near
Infrared Survey of the Southern Sky (DENIS; Epchtein et al. 1997). All of the targets have assigned spectral types between L6 and T5 with the exception of 2MASS 2208+29, which is an L2p. ${ }^{2}$ Properties of the targets appear in Table 1.

Observations were made in the $K_{s}$ band only, since many of the L dwarfs were too faint to be imaged in the $J$ band with this telescope. The targets were observed in a cyclic manner, each being observed a total of 2-5 times per night. During each observation, 18 images of the target were taken on a $3 \times 3$ square dither pattern (two images at each position). ${ }^{3}$ All exposures were $30 \mathrm{~s}$ in duration. Weather was mostly clear with some patchy clouds on several of the nights; October 2 was cloudy and yielded no usable data. Conditions were not always photometric, but for the following analysis only relative magnitudes between objects within an image were computed. Therefore, only if the average flux changed significantly during an 18 image set, or if clouds were too thick as determined by eye, were any image sets rejected. The average seeing for all nights was $1^{\prime \prime}-1$ ".8 (2-3 pixels).

The raw images were first dark-subtracted and flatfielded. Dome flats, twilight flats, and dark frames were obtained each night. Flat frames for each night were constructed by a median combination of the dome flats; since there appeared to be no residual structure in the images, the twilight flats were not used. Bad pixel masks were created to flag outliers and dead pixels in the flat field. Images were subsequently sky-subtracted using a median combination of the dithered science images and reduced images were then aligned using relative positions of the brightest objects in the field.

Aperture photometry was computed for all objects in each field using a 5 pixel aperture, with the sky value computed in a circular annulus. Due to the small field of view,

\footnotetext{
${ }^{2}$ This brown dwarf is classified as peculiar because it has $\mathrm{TiO}$ bands similar to an L4 dwarf, but $\mathrm{K}$ I lines similar to an L0 dwarf (Kirkpatrick et al. 2000). Note that we assume that all objects later than L6 are substellar; since the earlier objects have lithium detections (Kirkpatrick et al. 2000), we refer to all the targets as brown dwarfs.

${ }^{3}$ Due to the positioning of the one reference star in the field for 2MASS $0328+23$, this object was observed on a $2 \times 2$ square dither pattern with four images at each position.
}

TABLE 1

Properties of L and T Dwarf Targets

\begin{tabular}{|c|c|c|c|c|}
\hline Target Name & IAU Name & Type & $K_{s}(2 \mathrm{MASS})$ & Reference \\
\hline 2 MASS $2208+29$.. & 2MASSW J2208136+292121 & $\mathrm{L} 2 \mathrm{p}$ & $14.09 \pm 0.08$ & 1 \\
\hline 2MASS $0103+19 \ldots \ldots \ldots \ldots$. & 2MASSI J0103320+193536 & L6 & $14.15 \pm 0.07$ & 1 \\
\hline DENIS $0205-11 \ldots \ldots \ldots \ldots \ldots . . . .$. & DENIS-P J0205.4-1159AB & L7 & $13.0 \pm 0.2^{\mathrm{a}}$ & 2 \\
\hline 2MASS $0030-14 \ldots \ldots \ldots \ldots . . .$. & 2MASSW J0030300-145033 & L7 & $14.38 \pm 0.08$ & 1 \\
\hline 2 MASS $0328+23 \ldots \ldots \ldots \ldots \ldots$ & 2MASSI J0328426+230205 & L8 & $14.84 \pm 0.13$ & 1 \\
\hline 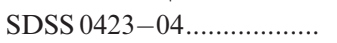 & SDSSp J042348.57-041403.5 & $\mathrm{T} 0^{\mathrm{b}}$ & $12.94 \pm 0.04$ & 3 \\
\hline SDSS $0151+12 \ldots \ldots \ldots \ldots \ldots \ldots$ & SDSSp J015141.69+124429.6 & $\mathrm{T} 1^{\mathrm{b}}$ & $15.09 \pm 0.19$ & 3 \\
\hline 2 MASS $2254+31 \ldots \ldots \ldots \ldots$ & 2MASSI J2254188+312349 & T5 & $14.83 \pm 0.14$ & 4 \\
\hline 2MASS $0559-14 \ldots \ldots \ldots \ldots . . .$. & 2MASSI J0559191-140448 & T5 & $13.61 \pm 0.05$ & 5 \\
\hline
\end{tabular}

Notes.- Spectral types from Kirkpatrick et al. (1999, 2000) and Burgasser et al. (2002c) unless otherwise noted. $K_{s}$ magnitudes are from 2MASS photometry.

a Magnitude is for the binary pair.

b Spectral type assigned by Geballe et al. 2002.

REFERENCES.- (1) Kirkpatrick et al. 2000; (2) Delfosse et al. 1997; (3) Geballe et al. 2002; (4) Burgasser et al. 2002c; (5) Burgasser et al. 2000. 
there was generally only one bright star available in each field. Therefore, differential photometry $(\Delta m)$ of targets was calculated with reference to a single bright star in the field (hereafter the comparison star). Differential photometry for all other stars in the field (hereafter field stars) was calculated with reference to this same comparison star. For two of the targets, only one other star (which is used as the comparison star) lies within the frame, so no field star is available. For the majority of other targets, field stars are comparable in brightness to the target.

\section{RESULTS}

We assume that no variation is taking place on the 10 minute timescale of each set of 18 dithered images. A relative magnitude difference $(\delta m)$ is calculated between the target and comparison star for each of the 18 images, by the method described in $\S 2$. The mean of these, $\Delta m=\overline{\delta m}$, is computed at each observation time interval, and the light curve constructed. One sigma error bars represent the error in the mean as computed from the scatter in $\delta m$ $\left(\sigma_{\Delta m}^{2}=\sigma_{\delta m}^{2} / N\right)$. Thus the error bars on $\Delta m$ are computed empirically from the data themselves, and no estimation of systematic errors has been made. This technique has the advantage that false detections associated with underestimating the errors present (excluding systematics) can be avoided. However, variations on short timescales (such that there is substantial change in the light curve in 10 minutes) will result in artificially large error bars, masking any variability signal present. In some cases, not all 18 images are included in $\Delta m$ because patchy clouds or changes in the seeing made accurate photometry of the faint targets impossible.

Light curves based on relative photometry for each target are shown in Figure 1, where the median value of $\Delta m$ has been subtracted from the data and an arbitrary constant added. Shown below each target is the light curve of a field star (computed using the same comparison star as for the target), when available. Note that a $420 \mathrm{hr}$ time span (from $\mathrm{AJD}=190.0-207.5)$ between set 1 and set 2 has been cut from the $x$-axis for clarity.

\subsection{Variability}

To determine whether any of the targets show evidence for variability, we define a robust median statistic:

$$
\eta=\sum_{i=1}^{N}\left|\frac{\Delta m_{i}-\operatorname{median}(\Delta m)}{\sigma_{i}}\right| .
$$

We choose to use a robust method to evaluate variability rather than the $\chi^{2}$ test because robust methods are much less sensitive to any non-Gaussian errors that may be present. ${ }^{4}$ We define the reduced $\eta$ to be $\tilde{\eta}=\eta / d$, where $d$ is the number of degrees of freedom. If the data are not varying, i.e., the intrinsic underlying distribution is a constant value, then the expected value of $\tilde{\eta}$ is less than one. A value of $\tilde{\eta} \gtrsim 1$ indicates that an object is likely to be variable. To quantify this likelihood, we ran Monte Carlo simulations.

\footnotetext{
${ }^{4}$ As a consistency check, we have also done a $\chi^{2}$ analysis following Bailer-Jones \& Mundt $(1999,2001)$, with very similar results to our robust method.
}

The significance of a given $\eta$ is determined by replacing the data with random noise (with a mean of zero and a standard deviation of one) and computing the resulting $\eta_{r}$. This process is repeated 1000 times; the number of times that these random $\eta_{r}$ are smaller than the real $\eta$ determines the confidence level. For example, if 980 of the random $\eta_{r}$ are smaller than $\eta$, then $\eta$ has a confidence of $98 \%$. We take a confidence above $95 \%$ to indicate significant evidence for variability.

The results of the robust analysis - the values of $\tilde{\eta}$ and the confidence (conf.) for each of the targets - are presented in Table 2. For comparison, variability results for the field stars are also shown. Note that if a comparison star were responsible for the variability seen in a target light curve, we would expect the field star to also show variability. The variability analysis is done for set 1 and set 2 separately, as well as for the combined set. Detections, i.e., those objects with conf. greater than $95 \%$, are shown in italics. Detections of which we are less confident for some reason (see $\S 3.1 .2$ ) are labeled possible detections and left in plain type.

The light curves were also examined to determine if there were any statistically significant changes in target brightness between set 1 and set 2 . The two sets were compared using the Student's $t$-statistic and distribution function. None of the targets showed a statistical difference between the data sets with a confidence above $95 \%$.

In order to compare the results for different objects, it is necessary to know the minimum amplitude at which variations could have been detected in each light curve. We choose detection limit of $99 \%$ because we want a stringent restriction on possible variations in the field star light curves and thus comparison star variability. If variability were present at an amplitude above the detection limit, it almost certainly would be detected. Sinusoidal detection limits are determined by generating sinusoidal data with a given amplitude and a period of $4.0 \mathrm{hr}$, which is close to the rotational period expected for $\mathrm{L}$ dwarfs from rotational velocity measurements (Basri et al. 2000). Changing the period chosen for this analysis does not greatly affect the detection limits, although in general shorter periods yield slightly lower limits. The signal is sampled at time intervals matching the actual observations, and random errors with standard deviations equal to the true errors in the data are added. This process is repeated 100 times at each amplitude, and the fraction of times that the signal produces a confidence greater than $95 \%$ is recorded. The amplitude at which 99 of the 100 test light curves are flagged as a detection is the 99\% detection limit.

Detection limits for random variations are determined in a similar way. Rather than a sinusoid, a series of random numbers with zero mean are generated, for which the amplitude is given by the largest peak-to-peak spread. While we would not actually expect our targets to vary in a random manner, we consider randomly varying light curves as an extreme case of nonsinusoidal variability. We note that it is generally easier to detect a sinusoidal variation than a random one, and the detection limits in set 2 are usually higher than for set 1 , due to the smaller number of data points in set 2 .

The 99\% detection limits and approximate amplitudes of variability, where applicable, for targets and field stars are listed in Table 3. For detections and possible detections, approximate peak-to-peak amplitudes are given in magnitudes, with uncertainties. Peak-to-peak amplitudes range 


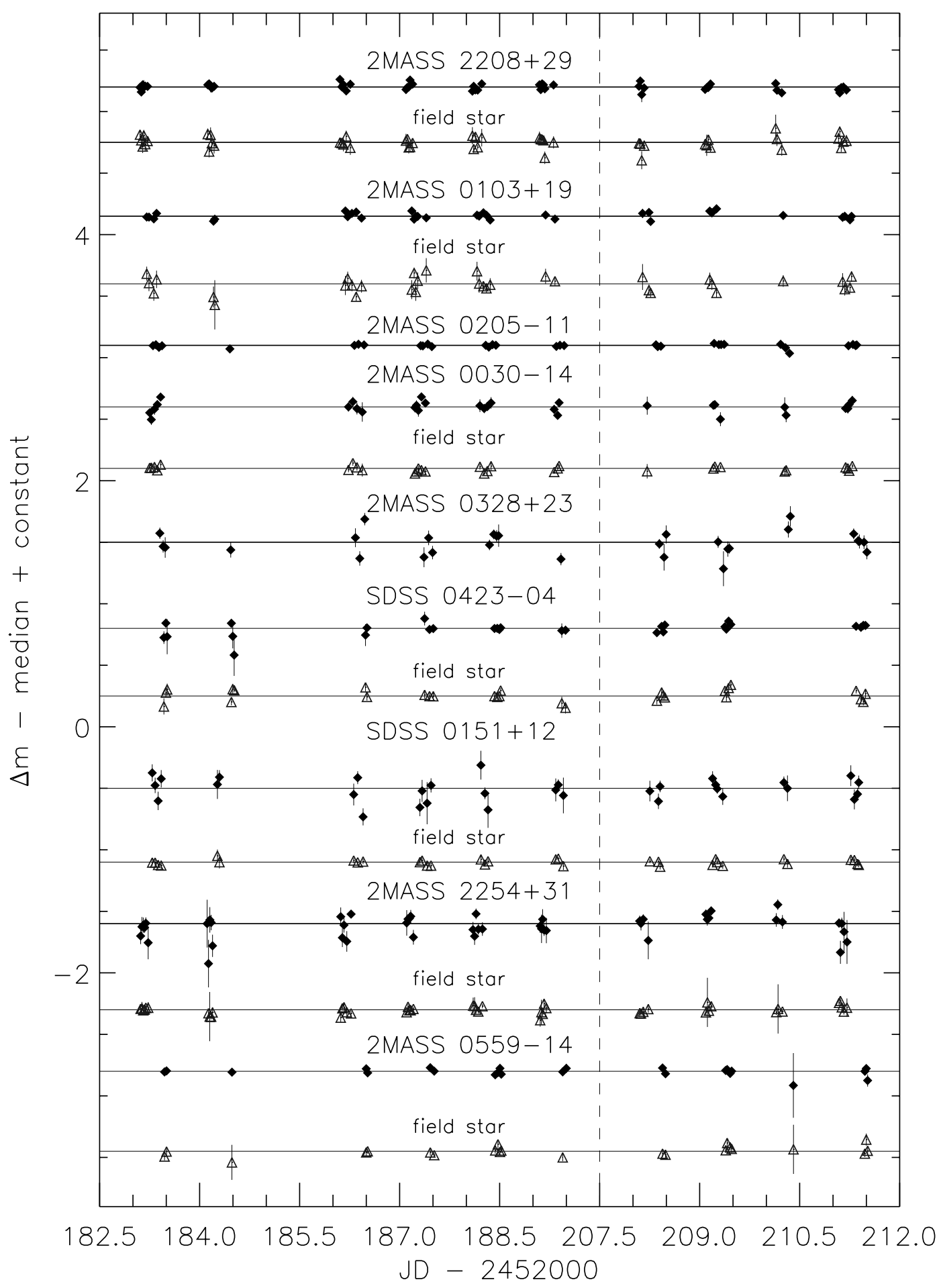

FIG. 1.-Relative photometry for all targets and field stars. Target data appear as filled diamonds and field star data as open triangles. For some targets, no field star is available. Note the break at JD =2,452,207.5 (dashed line), where $420 \mathrm{hr}$ between the two data sets have been removed for clarity.

from 0.10 to $0.48 \mathrm{mag}$, corresponding to approximate fractional deviations from the mean flux of $5 \%$ to $25 \%$. The uncertainty in the peak-to-peak amplitude is determined by adding in quadrature the $1 \sigma$ errors of the two data points used to determine the amplitude (the highest and lowest points in the light curve). Detection limits for both sinusoidal and random variations are given as peak-to-peak amplitudes (again in magnitudes) for all targets and field stars.

\subsubsection{Discussion of Individual Objects: Detections}

2MASS 0030-14: A confidence of 98\% makes this L7 dwarf a fairly strong detection in set 1 , but it is only a marginal detection (conf. $=95 \%$ ) in the combined set (see Table 2 ). An examination of the light curve, combined with the fact that the detection limit in set $2(0.22 \mathrm{mag})$ is greater than the amplitude in set $1(0.19 \mathrm{mag})$, makes it apparent that set 
TABLE 2

RESUltS OF VARIABILITY ANALYSIS

\begin{tabular}{|c|c|c|c|c|c|c|c|}
\hline \multirow[b]{2}{*}{ TARGET NAME } & \multirow[b]{2}{*}{ SPECTRAL TyPe } & \multicolumn{2}{|c|}{ SET 1} & \multicolumn{2}{|c|}{ SET 2} & \multicolumn{2}{|c|}{ Combined Set } \\
\hline & & $\tilde{\eta}$ & Conf. $(\%)$ & $\tilde{\eta}$ & Conf. $(\%)$ & $\tilde{\eta}$ & Conf. $(\%)$ \\
\hline 2 MASS $2208+29 \ldots \ldots \ldots \ldots . . .$. & $\mathrm{L} 2 \mathrm{p}$ & 0.76 & 34 & 0.82 & 54 & 0.79 & 44 \\
\hline 2 MASS $0103+19 \ldots \ldots \ldots \ldots .$. & L6 & 0.88 & 69 & 1.48 & $>99^{a}$ & 1.06 & $98^{\mathrm{a}}$ \\
\hline DENIS $0205-11 \ldots \ldots \ldots \ldots \ldots . . . .$. & L7 & 0.68 & 18 & 1.00 & 87 & 0.80 & 48 \\
\hline 2 MASS $0030-14 \ldots \ldots \ldots \ldots \ldots . .$. & $L 7$ & 1.09 & 98 & 0.79 & 33 & 1.00 & 95 \\
\hline 2 MASS $0328+23 \ldots \ldots \ldots \ldots .$. & L8 & 1.43 & $>99^{\mathrm{b}}$ & 1.03 & 88 & 1.21 & $>99^{\mathrm{b}}$ \\
\hline SDSS $0423-04 \ldots \ldots \ldots \ldots \ldots . . . . . . . .$. & T0 & 0.83 & 56 & 1.04 & 87 & 1.01 & $96^{\mathrm{c}}$ \\
\hline SDSS $0151+12 \ldots \ldots \ldots \ldots \ldots$ & $T 1$ & 1.15 & 99 & 0.87 & 64 & 0.99 & 95 \\
\hline $2 M A S S 2254+31 \ldots \ldots \ldots \ldots .$. & $T 5$ & 0.92 & 84 & 1.00 & 88 & 0.99 & 98 \\
\hline 2MASS 0559-14 ................ & $\mathrm{T} 5$ & 0.78 & 45 & 0.84 & 55 & 0.80 & 47 \\
\hline \multicolumn{8}{|c|}{ Field Stars } \\
\hline 2MASS $2208+29 \ldots \ldots \ldots \ldots . . . .$. & $\ldots$ & 0.84 & 62 & 0.86 & 62 & 0.84 & 66 \\
\hline 2 MASS $0103+19 \ldots \ldots \ldots \ldots \ldots . .$. & $\ldots$ & 0.83 & 57 & 0.95 & 75 & 0.84 & 67 \\
\hline DENIS 0205-11 ................... & $\ldots$ & $\ldots$ & $\ldots$ & $\ldots$ & $\ldots$ & $\ldots$ & $\ldots$ \\
\hline 2MASS 0030-14 ................ & $\ldots$ & 0.85 & 62 & 0.58 & 14 & 0.78 & 44 \\
\hline 2 MASS $0328+23 \ldots \ldots \ldots \ldots . .$. & $\ldots$ & $\ldots$ & $\ldots$ & $\ldots$ & $\ldots$ & $\ldots$ & $\ldots$ \\
\hline SDSS $0423-04 \ldots \ldots \ldots \ldots \ldots$ & $\ldots$ & 1.10 & 97 & 1.33 & 99 & 1.18 & $>99$ \\
\hline SDSS $0151+12 \ldots \ldots \ldots \ldots \ldots \ldots$ & $\ldots$ & 0.71 & 25 & 0.93 & 75 & 0.77 & 37 \\
\hline 2MASS $2254+31 \ldots \ldots \ldots \ldots$ & $\ldots$ & 0.81 & 51 & 0.97 & 82 & 0.82 & 59 \\
\hline 2MASS $0559-14 \ldots \ldots \ldots \ldots \ldots . .$. & $\ldots$ & 0.73 & 32 & 0.97 & 76 & 0.80 & 52 \\
\hline
\end{tabular}

Notes.-The variable $\tilde{\eta}$ is the reduced robust median statistic, and conf. is the percentage of times that an intrinsically nonvariable source with random errors had a smaller $\tilde{\eta}$. We take a confidence greater than $95 \%$ to indicate a variability detection; such detections are shown in italics. Possible detections (see notes and §3.1.2) are left in plain type. For two of the targets no field star was available.

${ }^{a}$ When the largest outlier is removed, these confidences drop below the $95 \%$ cutoff.

b No field star was available for 2 MASS $0328+23$, thus we are unable to confirm that variations are not due to the comparison star.

c Variability could be due to a variable comparison star. Note the variability detection in the field star section.

TABLE 3

Variability Amplitudes AND 99\% Detection Limits

\begin{tabular}{|c|c|c|c|c|c|c|}
\hline \multirow[b]{2}{*}{ TARGET NAME } & \multicolumn{2}{|c|}{ SET 1 (mag) } & \multicolumn{2}{|c|}{ SET 2 (mag) } & \multicolumn{2}{|c|}{ Combined Set (mag) } \\
\hline & Amp. & Det. Limit & Amp. & Det. Limit & Amp. & Det. Limit \\
\hline 2 MASS $2208+29 \ldots \ldots \ldots \ldots$ & $\ldots$ & $0.10(0.15)$ & & $0.17(0.16)$ & & $0.09(0.13)$ \\
\hline 2MASS $0103+19 \ldots \ldots \ldots \ldots$ & $\ldots$ & $0.09(0.13)$ & $0.10 \pm 0.02$ & $0.13(0.12)$ & $0.10 \pm 0.02$ & $0.08(0.11)$ \\
\hline DENIS $0205-11 \ldots \ldots \ldots \ldots . . .$. & $\ldots$ & $0.04(0.05)$ & $\ldots$ & $0.04(0.06)$ & $\ldots$ & $0.03(0.05)$ \\
\hline $2 M A S S 0030-14 \ldots \ldots \ldots \ldots \ldots$ & $0.19 \pm 0.11$ & $0.14(0.19)$ & $\ldots$ & $0.22(0.22)$ & $0.19 \pm 0.11$ & $0.12(0.20)$ \\
\hline 2 MASS $0328+23 \ldots \ldots \ldots \ldots$ & $0.32 \pm 0.07$ & $0.27(0.32)$ & $\ldots$ & $0.23(0.33)$ & $0.43 \pm 0.16$ & $0.21(0.29)$ \\
\hline SDSS $0423-04 \ldots \ldots \ldots \ldots \ldots$ & $\ldots$ & $0.14(0.18)$ & $\ldots$ & $0.08(0.10)$ & $0.30 \pm 0.18$ & $0.08(0.11)$ \\
\hline SDSS $0151+12 \ldots \ldots \ldots \ldots \ldots$ & $0.42 \pm 0.14$ & $0.25(0.41)$ & $\ldots$ & $0.30(0.33)$ & $0.42 \pm 0.14$ & $0.22(0.35)$ \\
\hline 2 MASS $2254+31 \ldots \ldots \ldots \ldots .$. & $\ldots$ & $0.21(0.33)$ & $\ldots$ & $0.32(0.30)$ & $0.48 \pm 0.20$ & $0.20(0.28)$ \\
\hline 2MASS $0559-14 \ldots \ldots \ldots \ldots \ldots$ & $\cdots$ & $0.09(0.11)$ & $\cdots$ & $0.20(0.20)$ & $\ldots$ & $0.07(0.11)$ \\
\hline \multicolumn{7}{|c|}{ Field Stars } \\
\hline 2MASS $2208+29 \ldots \ldots \ldots \ldots . .$. & $\cdots$ & $0.14(0.22)$ & $\cdots$ & $0.25(0.24)$ & $\cdots$ & $0.14(0.19)$ \\
\hline 2 MASS $0103+19 \ldots \ldots \ldots \ldots$ & $\ldots$ & $0.20(0.31)$ & $\ldots$ & $0.32(0.30)$ & $\ldots$ & $0.19(0.28)$ \\
\hline DENIS $0205-11 \ldots \ldots \ldots \ldots \ldots$ & $\ldots$ & & $\ldots$ & & $\ldots$ & \\
\hline 2MASS $0030-14 \ldots \ldots \ldots \ldots \ldots$ & $\cdots$ & $0.08(0.13)$ & $\ldots$ & $0.09(0.13)$ & $\ldots$ & $0.07(0.11)$ \\
\hline 2MASS $0328+23 \ldots \ldots \ldots \ldots$ & $\ldots$ & & $\ldots$ & & $\ldots$ & \\
\hline SDSS 0423-04 .................. & $0.17 \pm 0.06$ & $0.14(0.18)$ & $0.14 \pm 0.04$ & $0.14(0.17)$ & $0.19 \pm 0.05$ & $0.10(0.16)$ \\
\hline SDSS $0151+12 \ldots \ldots \ldots \ldots \ldots$ & $\ldots$ & $0.08(0.13)$ & $\ldots$ & $0.10(0.13)$ & $\ldots$ & $0.07(0.12)$ \\
\hline 2 MASS $2254+31 \ldots \ldots \ldots \ldots$ & $\ldots$ & $0.09(0.14)$ & $\ldots$ & $0.16(0.17)$ & $\ldots$ & $0.08(0.14)$ \\
\hline 2MASS $0559-14 \ldots \ldots \ldots \ldots . .$. & $\ldots$ & $0.12(0.17)$ & $\ldots$ & $0.16(0.20)$ & $\ldots$ & $0.10(0.15)$ \\
\hline
\end{tabular}

Notes.-Amplitudes are peak to peak and given in magnitudes. The uncertainty in the two data points used to compute the amplitude are added in quadrature to estimate the amplitude uncertainty. Detection limits are the peak-to-peak amplitude for which $99 \%$ of sinusoidal variations are detected. Numbers in parentheses are similar, but for random variations. 
2 could have been a detection were more data available. The 99\% detection limits for the field star ( 0.07 mag for sinusoidal variation and $0.11 \mathrm{mag}$ for random variations) are about $1 \sigma$ below the detected amplitude of variation for 2MASS $0030-14(\sim 0.19 \pm 0.11 \mathrm{mag})$. Furthermore, as we will see in $\S 3.2$, the variations in 2MASS 0030-14 appear to be close to sinusoidal. Since no such behavior is seen in the field star light curve, this strengthens our confidence that the variability is intrinsic to 2MASS $0030-14$.

SDSS 0151+12: This T1 dwarf is a detection in set 1 $(99 \%)$ and marginally in the combined set $(95 \%)$. The estimated amplitude $(0.42 \pm 0.14 \mathrm{mag})$ is well above the field star detection limits of $0.07 \mathrm{mag}$ (sinusoidal variations) and $0.12 \mathrm{mag}$ (random variations). Thus it is unlikely that the observed variability is due to the comparison star. No evidence for variability is seen in set $2($ conf. $=64 \%)$, even though the detection limits in set $2(0.30$ and $0.33 \mathrm{mag})$ are less than the set 1 amplitudes (they are, however, within $1 \sigma$ ). This suggests that the amplitude of variation may have decreased between the two sets. As with 2MASS 0030-14, evidence for periodic variations in $\S 3.2$ strengthens our confidence in this detection.

2MASS 2254+31: The T5 dwarf 2MASS $2254+31$ is a detection in the combined set $(98 \%)$, primarily due to an increase in flux during the second and third nights of set 2. This two night trend is not seen in the field star light curve or that of any of the other targets, although there are similar deviations in 2MASS $0328+23$ and 2MASS $0205-11$ on the third night only. There is no detection in set 1 , despite the sinusoidal detection limit $(0.21 \mathrm{mag})$ being more than $1 \sigma$ lower than the estimated amplitude in set $2(0.48 \pm 0.20)$. Given that the random variation limit $(0.33)$ is less than $1 \sigma$ below the amplitude, it seems likely that the variations are nonsinusoidal.

\subsubsection{Discussion of Individual Objects: Possible Detections}

2MASS 0103+19: This L6 dwarf is detected as variable in set 2 and the combined set, due to a short rise in flux at around JD $=2,452,209.3$. When the highest point is removed, 2MASS $0103+19$ drops just below the detection limit (conf. $=92 \%$ in set 2 and $89 \%$ for the combined set). Detection limits for the field star $(\sim 0.2$ mag sinusoidal, $\sim 0.3$ mag random) are substantially greater than the observed peak-to-peak amplitude of variability $(\sim 0.1 \pm 0.02 \mathrm{mag})$, so we are unable to rule out the possibility that the detected variability may be due to the comparison star. We note, however, that if we use the field star as the comparison star, we still find variability in 2MASS $0103+19$ with conf. $=95 \%$ for set 2 . The high detection limits and larger observed scatter in the field star light curve are due to the fact that the field star is almost 1 mag fainter than 2MASS $0103+19$. Given the uncertainties present, we label 2MASS $0103+19$ a possible detection.

2MASS 0328+23: 2 MASS $0328+23$, an L8 dwarf, has one of the largest amplitudes of variability for the detections here $(\sim 0.43 \pm 0.16 \mathrm{mag}$ for the combined set) and a very high confidence (greater than $99 \%$ in set 1 and the combined set). The light curve (Fig. 1) illustrates that the scatter in the data is much greater than the error bars. Since there is no field star available, however, it is impossible to tell whether this variability is intrinsic to the brown dwarf or to the comparison star.

SDSS 0423-04: SDSS 0423-04 is a detection in the combined set with a confidence of $96 \%$. It is clear, however, from the field star values in Table 2 that either the comparison star or field star is variable in this case (conf. $=97 \%$ for set 1 and conf. $>99 \%$ for set 2 and the combined set). When we examine the aperture photometry (i.e., the apparent magnitude $m$, not $\Delta m$ ) of the target, the field star and the comparison star, we find that the trends in the field star light curve that cause it to be flagged as variable seem to be caused by the field star. Thus we believe that it is likely the field star, not the comparison star, which is variable. If the field star is used as the comparison star, SDSS 0423 becomes a detection in set 2 only. It is still unclear whether or not SDSS $0423-04$ is actually the source of these variations, thus it is a possible detection.

\subsection{Periodicity}

When significant evidence for variability is found, we check for the existence of periodic variations by constructing the Lomb-Scargle-weighted periodogram for unevenly sampled data (Woodward 1992). Given the large gap between the data sets, we choose to analyze the set 1 and set 2 data independently. In computing the periodogram, we allow for a maximum period of $150 \mathrm{hr}$ (approximately equal to the observational baseline for set 1) and a minimum period of $1.0 \mathrm{hr}$ (approximately the Nyquist sampling limit). Peaks in the periodogram indicate how well a sinusoid with a given period fit the data. The statistical significance of these peaks are determined in the following way: the data are shuffled into random order, keeping the same observation times. This shuffling is repeated 1000 times, and the highest periodogram peak for each recorded. The percentage of shuffled peaks below a given value determines that value's significance.

Table 4 gives the results of the periodogram analysis for variable and possibly variable targets. The best period, corresponding to the highest peak in the periodogram, is given in hours followed by the confidence that the peak is not due to noise or sampling effects, as determined by shuffling. There are no detected periods with a confidence greater than $95 \%$ for any of the objects; in fact only two of the targets, 2MASS 0030-14 and SDSS 0151+12, have periodogram peaks above the $68 \%(\approx 1 \sigma)$ level. Figures 2 and 3 show the periodograms of these two targets. The top plots show the original periodograms; in the bottom plots, each point has been replaced by its corresponding confidence level. Figures 4 and 5 show similar plots for the field stars. Were the periodic behavior observed intrinsic to the comparison star rather than the target, the field star should also exhibit periodic behavior. Thus a lack of strong peaks in the field star periodogram indicates that the tentative periodicity is intrinsic to the target.

Another way to distinguish real peaks from spurious ones, apart from our method of shuffling, is to deconvolve the sampling window function from the periodogram. The Fourier transform of the true light curve is convolved with the sampling window function during observation, causing false peaks and structure in the periodogram. The analytic window function in this case is the Fourier transform of a sum of delta functions located at the sampling times. When the data are deconvolved using a maximum likelihood routine, it is found that any significant peaks present in the deconvolved periodogram almost always correspond to the strongest peak in the original periodogram. For this reason, we 
TABLE 4

Results of Lomb-Scargle Periodogram Analysis

\begin{tabular}{|c|c|c|c|c|}
\hline \multirow[b]{2}{*}{ TARgEt NAME } & \multicolumn{2}{|c|}{ SET 1} & \multicolumn{2}{|c|}{ SET 2} \\
\hline & Period (hrs) & Confidence $(\%)$ & Period (hrs) & Confidence $(\%)$ \\
\hline 2MASS $0103+19 \ldots \ldots \ldots \ldots$ & 1.95 & 42 & 2.24 & 58 \\
\hline $2 M A S S 0030-14 \ldots \ldots \ldots \ldots \ldots$ & 1.38 & 90 & 10.7 & 10 \\
\hline 2 MASS $0328+23 \ldots \ldots \ldots \ldots$ & 3.87 & 5 & 2.10 & $<1$ \\
\hline SDSS $0423-04 \ldots \ldots \ldots \ldots \ldots \ldots$ & 1.39 & $<1$ & 1.10 & 11 \\
\hline SDSS $0151+12 \ldots \ldots \ldots \ldots \ldots$ & 2.97 & 89 & 1.43 & 15 \\
\hline 2 MASS $2254+31 \ldots \ldots \ldots \ldots$ & 1.21 & 4 & 2.65 & 9 \\
\hline \multicolumn{5}{|c|}{ Field Stars } \\
\hline 2MASS $0103+19 \ldots \ldots \ldots \ldots$ & 2.14 & 10 & 3.94 & 6 \\
\hline 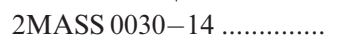 & 4.96 & 45 & 1.76 & 3 \\
\hline 2MASS $0328+23 \ldots \ldots \ldots \ldots$ & $\ldots$ & $\ldots$ & $\ldots$ & $\ldots$ \\
\hline SDSS $0423-04 \ldots \ldots \ldots \ldots \ldots$ & 1.62 & 46 & 1.00 & 99 \\
\hline SDSS $0151+12 \ldots \ldots \ldots \ldots \ldots \ldots$ & 1.17 & 34 & 2.10 & 5 \\
\hline 2MASS $2254+31 \ldots \ldots \ldots \ldots$ & 1.17 & 9 & 3.87 & 3 \\
\hline
\end{tabular}

NoTes.- Only targets that were found to be variable or possibly variable (see Table 2) are shown.

choose to quantify the periodogram peaks in terms of their confidence level (as in Figures 2 and 3), which gives us a better basis for comparing results.

The light curves of the two periodic detections are phased to their best period as determined by the periodogram and plotted in the top panels of Figures 6 and 7. Note that although in both cases the best period is determined from the set 1 data only, all the data have been phased to this period in the figures. In the bottom panels are the field star light curves, phased to the same period as the target (not their own best period). As a further check on the believability of these periods, Figures 8 and 9 show each day of observation denoted by a different symbol.

The other objects in Table 4 were also phased to their best period from the periodogram and checked by eye, but none displayed any obviously periodic behavior. As Bailer-Jones

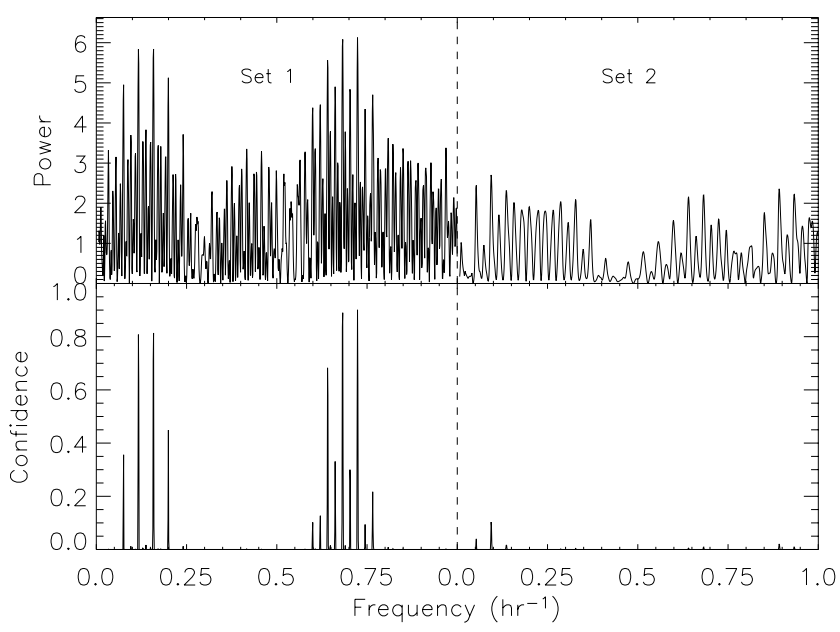

FIG. 2.-Periodogram for 2 MASS $0030-14$, for both set 1 and set 2 . The top plot is the original Lomb-Scargle periodogram. The bottom plot is the same periodogram scaled to confidence units. Thus a value of 0.8 indicates $80 \%$ confidence that a peak is not due to sampling effects or random noise. The dashed line denotes the separation between set 1 and set 2 periodograms.
\& Mundt (2001) note, the light curves of objects that are variable due to patchy clouds will not always look sinusoidal. Furthermore, it is much more difficult to detect periodic behavior than it is to detect variability. Given that none of the variability detections have extremely high confidence, we cannot rule out the presence of periodic variations in the other variable, or possibly variable targets.

\subsubsection{Discussion of Individual Objects: Possible Detections}

2MASS 0030-14: There are two close peaks (at 1.38 and $1.46 \mathrm{hr}$, or 0.72 and $0.68 \mathrm{hr}^{-1}$ ) in the 2MASS $0030-14$ periodogram at approximately the same $(90 \%)$ confidence level (Fig. 2). Either of these could be considered the best period; we have chosen to use the $1.46 \mathrm{hr}$ period. The next highest peak, at $6.67 \mathrm{hr}\left(0.15 \mathrm{hr}^{-1}\right)$ and $85 \%$ confidence, also shows possible periodic behavior, but not as clearly as for $1.46 \mathrm{hr}$. Note that the field star periodogram (Fig. 4) never exceeds $50 \%$ confidence. The light curve of 2MASS $0030-14$, phased to $1.46 \mathrm{hr}$ (Fig. 6), is not exactly sinusoidal, but the periodic nature is evident. The amplitude of the curve indicates a fractional deviation from the mean flux of

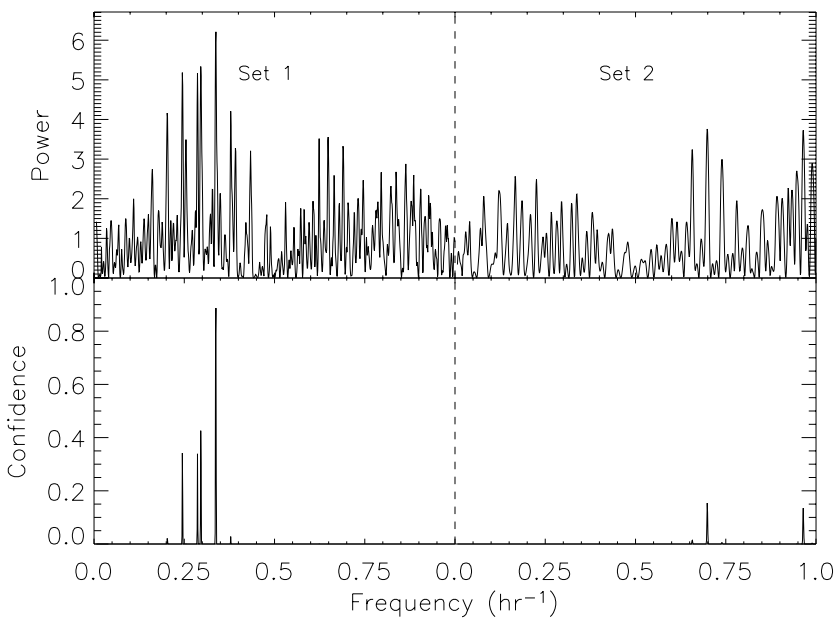

FIG. 3.-Periodogram for SDSS $0151+12$. See Fig. 2 for explanation. 


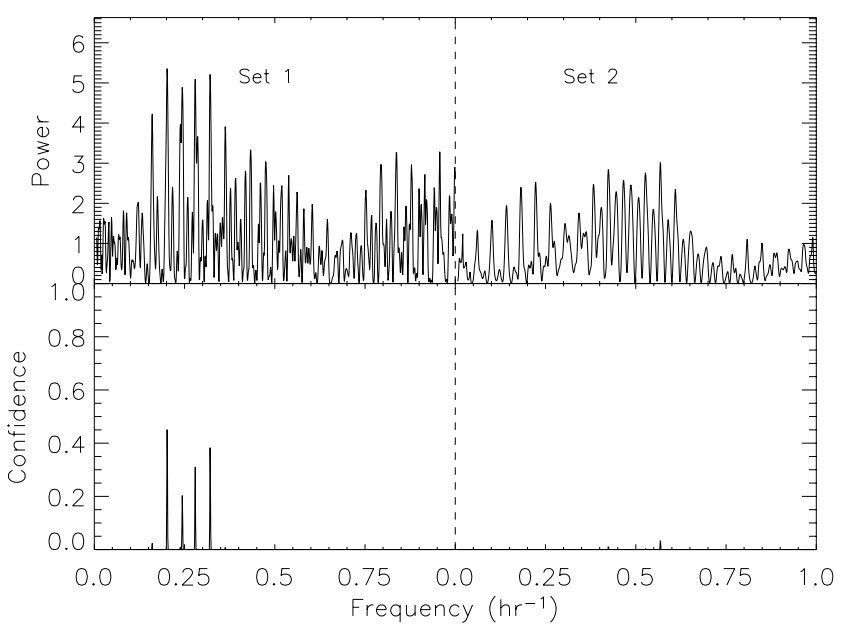

FIG. 4.-Periodogram for the field star of 2MASS 0030-14. See Fig. 2 for explanation.

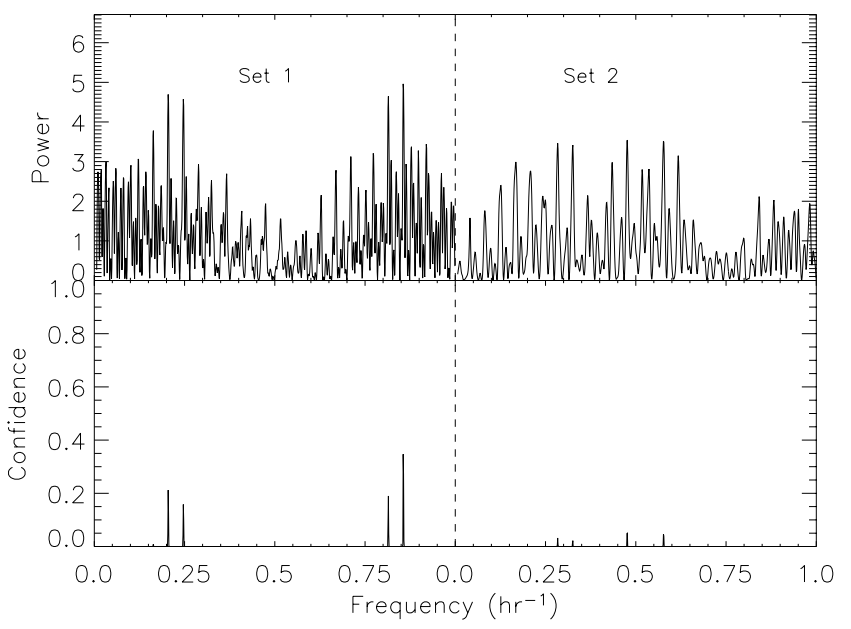

FIG. 5.-Periodogram for the field star of SDSS $0151+12$. See Fig. 2 for explanation.

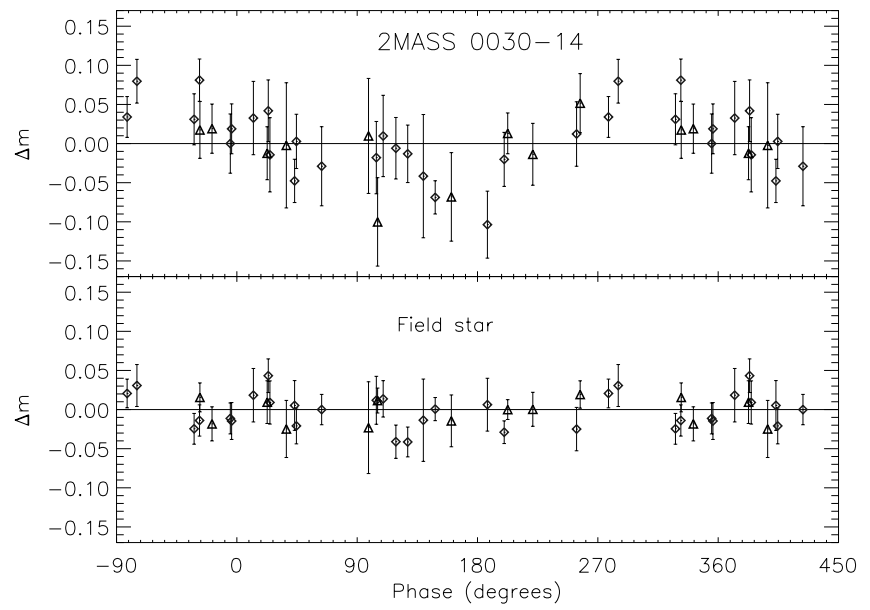

FIG. 6.-Light curve of 2MASS 0030-14, phased to a period of $1.46 \mathrm{hr}$. In total, 1.5 periods are plotted. Diamonds indicate data from set 1 , and triangles data from set 2 . The field star light curve is also phased to $1.46 \mathrm{hr}$ for comparison.

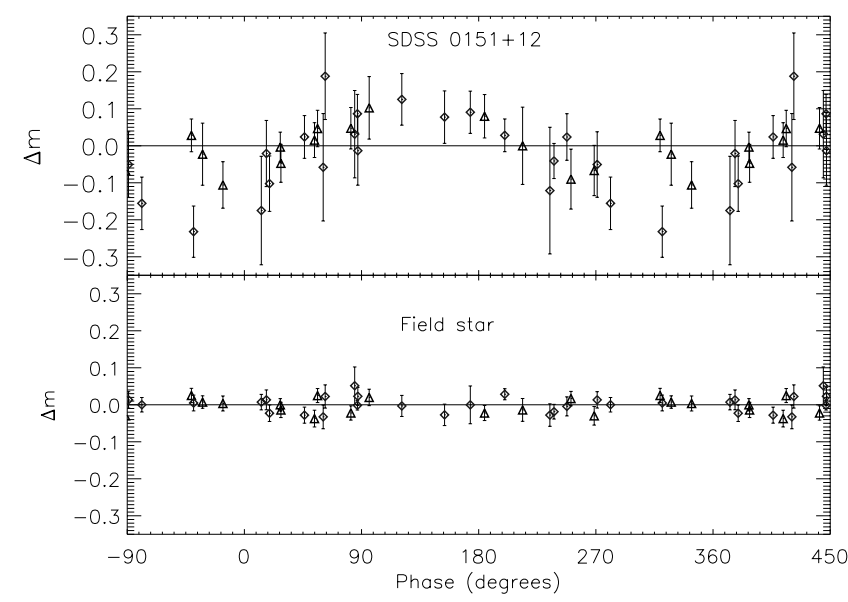

FIG. 7.- - Light curve for SDSS $0151+12$ phased to a period of $2.97 \mathrm{hr}$. See Fig. 6 for explanation. Here the data for set 2 have been shifted by $110^{\circ}$ of phase to visually align them with the set 1 data. Note the rise in set 2 around $330^{\circ}$, which may indicate that either there is an extra bump in the set 2 light curve, or the period for set 2 is not the same as that for set 1 .

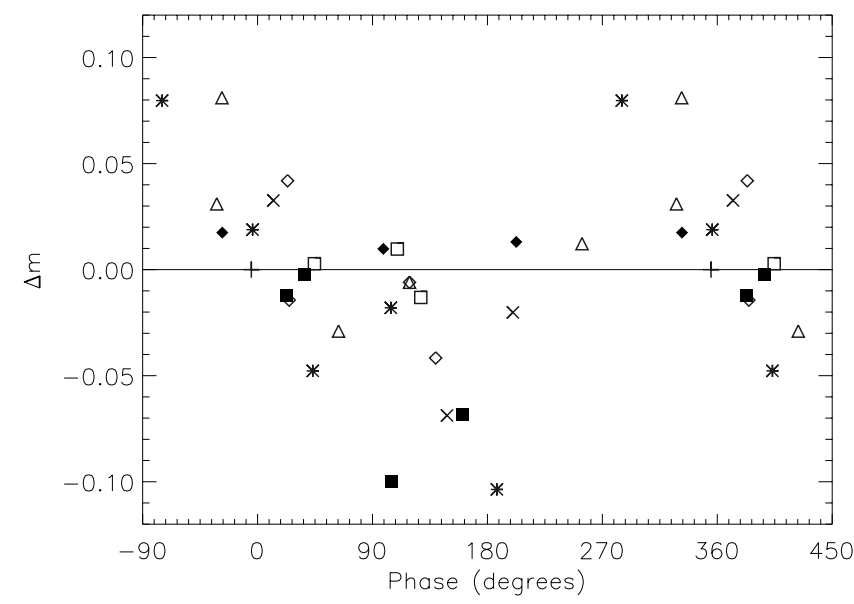

FIG. 8.-Phased light curve of 2MASS 0030-14, similar to Fig. 6, but here each day of observation is denoted by a different symbol. The filled symbols correspond to days in set 2 .

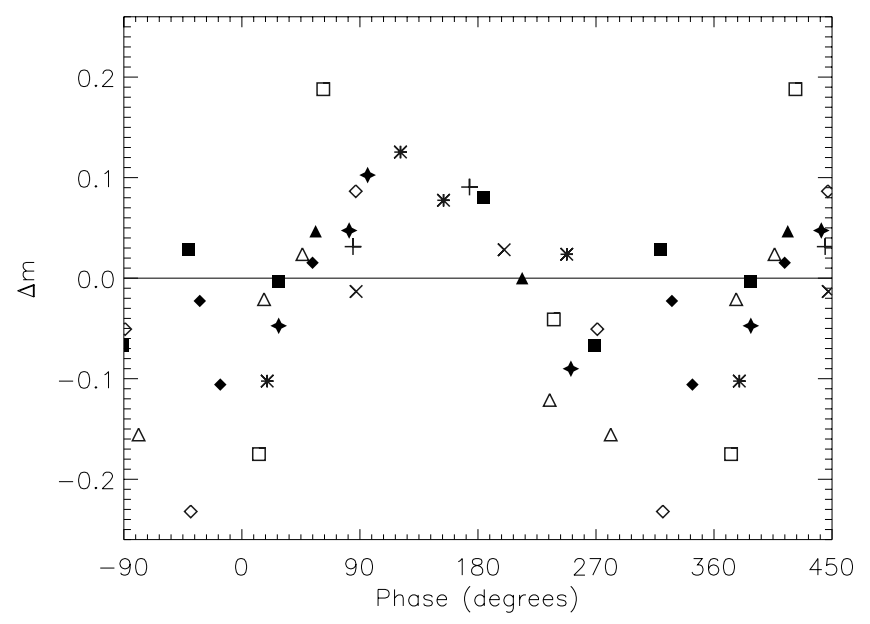

FIG. 9. - Phased light curve of SDSS $0151+12$, plotted by day. See Fig. 8 for explanation. 
$\sim 10 \%$. The periodic behavior is indeed intrinsic to the brown dwarf and not to the comparison star, as can be seen by examining the field star light curve phased to the same period. In addition, note that in Figure 8, the data from each day follow approximately the same curve without any systematic trends, supporting the conclusion that the observed periodicity is real. In Figure 6, the data from set 1 (diamonds) and set 2 (triangles) match well without any phase shift between them; if this is the correct period, it is probably stable over the length of our observations.

SDSS 0151+12: The periodogram for SDSS $0151+12$ (Fig. 3) has only one significant peak, at a period of $2.96 \mathrm{hr}$ $\left(0.34 \mathrm{hr}^{-1}\right)$ and a confidence of $89 \%$. The phased light curve (Fig. 7) shows a nearly sinusoidal curve for the set 1 data. The set 2 data, however, have an extra peak around $330^{\circ}$. This peak is present in at least two of the nights in set 2, as is evident from Figure 9. We propose three possible reasons for the slight mismatch between set 1 and set 2: the light curve changed between the two sets with an increase in flux appearing around $330^{\circ}$, the period of variation changed between the two sets so that the phased light curve no longer shows one period, or the derived period of variation is incorrect. It is clear that a change in the light curve could cause the set 2 data to have a smaller overall amplitude and help explain why SDSS $0151+12$ is not a detection in set 2 . The amplitude of the set 1 data corresponds to a fractional deviation from the mean flux of $\sim 20 \%$. Note that the set 2 data have been shifted by $110^{\circ}$ of phase in order to visually align them with the set 1 data. Such a phase offset is not unexpected; even a small error in the period can cause large phase offsets because the observational baseline is long compared with one period. For example, if we make a $1 \%$ error in a $3 \mathrm{hr}$ period, the $420 \mathrm{hr}$ time span between set 1 and set 2 will introduce a phase offset of $500^{\circ}$.

\section{DISCUSSION}

Our result, that at least one-third of our targets are variable (and as many as two-thirds, if possible detections are accounted for), is consistent with previous surveys of $\mathrm{L}$ dwarf variability. Bailer-Jones \& Mundt (2001) find seven of their 10 early L dwarfs to be variable, Gelino et al. (2002) find seven of 18 variable, and Clarke et al. (2002a) find two of four variable. However, Bailer-Jones \& Lamm (2003) fail to find variability at the $0.04 \mathrm{mag}$ level in the $J$ and $K^{\prime}$ bands for three early L dwarfs. Given these statistics and our current results, there is no evidence that objects near the $\mathrm{L} / \mathrm{T}$ boundary are more likely to be variable than earlier L dwarfs. It is clear, however, that variability does exist in even these very cool objects.

Given that most of the previous studies were conducted in the $I$ band, it is impossible to quantitatively compare detection limits with our $K_{s}$-band observations. We note, however, that the detection limits of Bailer-Jones \& Lamm (2003) are well below our observed amplitudes. The fractional amplitudes of variation in the $I$ band for most early $\mathrm{L}$ dwarfs is small, with few above the 1\%-2\% level (Clarke et al. 2002). Our variable objects show much larger deviations, but again such comparison across wave bands are difficult to quantify. Further studies at infrared wavelengths will need to be done before we can meaningfully compare either the fraction of variable objects or the variability amplitudes of early $\mathrm{L}$ dwarfs and $\mathrm{L} / \mathrm{T}$ transition objects.

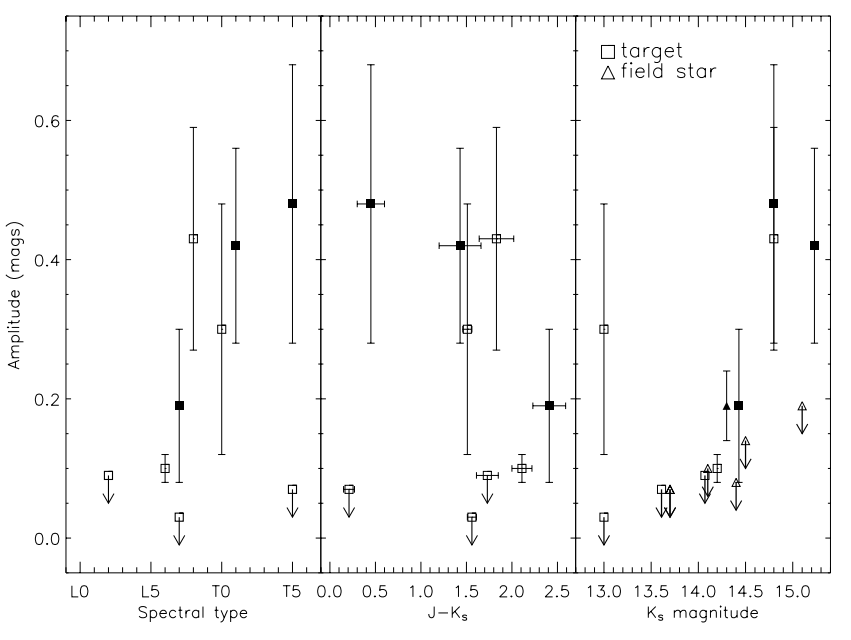

FIG. 10.-Variability amplitudes and detection limits from Table 3 vs. spectral type, $J-K_{s}$ color, and $K_{s}$ magnitude. Left: Squares with error bars indicate variability amplitudes for detections (filled squares) and possible detections (open squares). For nondetections, the $99 \%$ sinusoidal detection limits are denoted by open squares with arrows. Center: $J-K_{s}$ values and errors are from references in Table 1. Right: Similar to left panel, but here field star amplitudes and detection limits are included as triangle symbols. The $K_{s}$ magnitude of a field star is computed from the average differential photometry: $\left(K_{s}\right)_{\text {field }}=\left(K_{s}\right)_{\text {target }}-\overline{\Delta m_{\text {target }}}+\overline{\Delta m_{\text {field }}}$.

In light of the cloud-clearing model fit (Burgasser et al. $2002 b$ ), we might expect the photometric signal to be rotationally modulated. Therefore, if the signal were due to a single atmospheric feature, the period of variation would be equal to the rotational period. Were the $1.5 \mathrm{hr}$ variation of 2MASS $0030-14$ a true measurement of the rotational period, the rotational velocity of this object would lie at the high end of observations (Basri et al. 2000), but it is well below breakup velocity for any $M>0.01 M_{\odot}$. The $3.0 \mathrm{hr}$ variation of SDSS $0151+12$ would also translate to fairly rapid rotation. In the context of this picture, we might also expect a trend in variability with spectral type, although the relationship would not be linear. If cloud breakup occurs at the transition from $\mathrm{L}$ to $\mathrm{T}$ dwarfs, the variability should peak at that point, falling off toward both mid $\mathrm{L}$ and mid $\mathrm{T}$ dwarfs.

In Figure 10, we plot amplitudes of variability and detection limits as a function of spectral type (left), $J-K_{s}$ color (center), and $K_{s}$ magnitude (right). There is no clear visual evidence to suggest that $\mathrm{L} / \mathrm{T}$ boundary objects are more likely to be variable than later or earlier type objects. To test for a statistical correlation between spectral type and variability amplitude, we compute the Spearman rank correlation coefficient $\left(-1 \leq \rho_{s} \leq 1\right)$ and significance value $(0 \leq \zeta \leq 1)$. A value of $\rho_{s}=1$ implies perfect correlation, and the smaller $\zeta$, the more significant the result. Note that for the following, nondetections are assumed to have variability amplitudes of zero. ${ }^{5}$ For amplitude versus spectral type, we find $\rho_{s}=0.48$, which corresponds to a significance of $\zeta=0.19$ and a two-sided null hypothesis probability $\left(P_{\text {null }}\right)$ of about $18 \%$. Thus there is no strong evidence for a correlation between variability amplitude and spectral type.

\footnotetext{
${ }^{5}$ Another possibility is to let nondetection amplitudes equal their detection limits. We find that the two methods give very similar results.
} 
When we apply the rank correlation test to variability amplitude versus $J-K_{s}$ color, we find no correlation $\left(P_{\text {null }}=80 \%\right)$, as verified by an examination of Figure 10. A similar comparison of amplitude versus $K_{s}$ mag, however, yields values of $\rho_{s}=0.71, \zeta=0.03$, and $P_{\text {null }}=4 \%$, thus the two properties are correlated with $\sim 96 \%$ confidence. It is unclear why magnitude and variability would be correlated, given that apparent magnitude is primarily a function of distance, not physical properties. While this may seem to indicate a problem with our analysis, we note that no such trend is seen for the field stars (see Fig. 10), which have comparable $K_{s}$ magnitudes. This discrepancy suggests that there may be a hidden dependency causing these correlations. If, for example, all the targets were at the same distance, we would expect apparent magnitude and spectral type to be well correlated. Curiously, if we neglect the three brightest objects, there are strong correlations $\left(P_{\text {null }} \leq 7 \%\right)$ between amplitude and spectral type, amplitude and $K_{s}$ magnitude, and magnitude and spectral type. We suspect that complex selection effects involving target selection, apparent magnitude, and spectral type are complicating these results. A larger sample is needed to disentangle these dependencies and determine if there are any trends of variability frequency or amplitude with spectral type.

As Gelino et al. (2002) point out, if holes in the cloud deck are responsible for photometric variability in brown dwarfs, we might expect the $J-K_{s}$ colors of variable objects to be bluer than those of nonvariable objects (which are assumed to have uniform cloud coverage) for a given spectral type. In Figure 11, we plot $J-K_{s}$ versus spectral type for our targets, as well as the average $J-K_{s}$ as a function of spectral type from Kirkpatrick et al. (2000) and Burgasser et al. (2002c). Unfortunately, there are so few $J-K_{s}$ measurements for L9-T2 objects that there are no average colors available. From this plot, there is no indication that variable objects have bluer $J-K_{s}$ colors compared with nonvariable objects. There is also no evidence that variable objects are bluer than the average $J-K_{s}$ for their spectral type. If, however, at least some nonvariable brown dwarfs do not vary because the holes or structure in their atmosphere is more

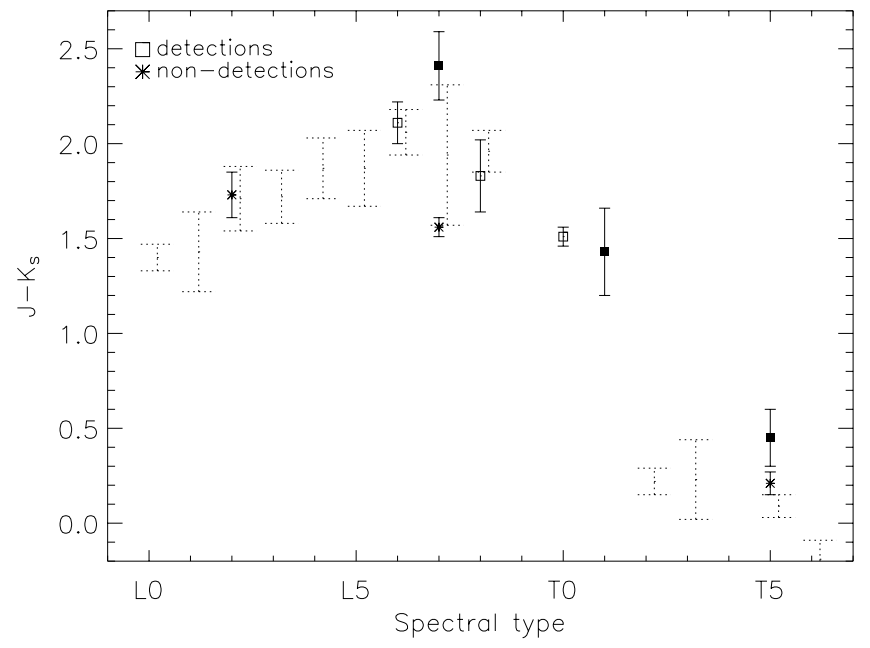

FIG. 11. $-J-K_{s}$ colors of targets as a function of spectral type. Filled squares indicate detections, open squares possible detections, and stars nondetections. Dotted bars are average 2MASS $J-K_{s}$ values, with errors, from Kirkpatrick et al. (2000) and Burgasser et al. (2002c). uniform (e.g., many small holes), this correlation would not be so simple.

It has recently been suggested that any observed IR variability of brown dwarfs may be due entirely to secondorder telluric extinction effects (Bailer-Jones \& Lamm 2003). The unique spectral energy distribution of brown dwarfs coupled with wavelength-dependent atmospheric extinction may cause different fractional decreases in intensity with air mass across a broadband filter. This effect could cause the magnitude difference between brown dwarf and comparison star to change over the course of observation, creating an artificial variability signal. To check for such a bias, we examined plots of $\Delta m$ versus air mass for each object. Again applying the rank correlation test, we find that for most objects $P_{\text {null }} \geq 60 \%$. The lowest null probability, for SDSS $0151+12$, is $22 \%$, but when the highest airmass point is removed (which does not affect any other results), $P_{\text {null }}$ rises to greater than $40 \%$. Therefore, we find no evidence that $\Delta m$ is correlated with air mass for any of the targets and argue that changing air mass is not responsible for the observed variability. Furthermore, it seems unlikely that atmospheric effects could produce a periodic signal, as is seen here in two objects. Although we recognize that extinction effects could possibly complicate some of our detections, we remain confident of our general results.

\section{SUMMARY}

We have obtained $K_{s}$-band light curves for nine late L and early $\mathrm{T}$ dwarfs. Results indicate that three show significant evidence for variability, and several more show evidence for possible variability, with approximate fractional deviations from the mean flux of $5 \%$ to $25 \%$ (peak-to-peak amplitudes of 0.1-0.5 mag). Two objects, 2MASS 0030-14 (L7) and SDSS 0151+12 (T1), have marginally significant peaks in the Lomb-Scargle periodogram. To these objects, we assign tentative periods of 1.5 and $3.0 \mathrm{hr}$, respectively. In the case of SDSS $0151+12$, it seems likely that either the period of variation or the shape of the light curve changed over the 29 day observational baseline.

While it is clear that variability exists in these cool objects, the source of variability is still unknown. As noted in $\S 1$, it seems unlikely that magnetic activity could account for the variability seen here. We see no significant trend of variability with spectral type, which might be expected were the breakup of cloud decks responsible. Similarly, there is no evidence that variable objects are bluer than nonvariable objects of the same spectral type. Time-resolved spectroscopic measurements of variable targets might be able to conclusively determine whether the variability seen in this survey is due to structure in the cloud coverage or some other phenomenon.

The authors are grateful to Antonin Bouchez, JeanLuc Margot, and the Palomar Observatory staff for their time and help in obtaining these observations. We also thank the referee for helpful comments on the manuscript. M. L. E. acknowledges support from a NSF Graduate Research Fellowship. A. J. B. acknowledges support by NASA through Hubble Fellowship grant HST-HF-01137.01 awarded by the Space Telescope Science Institute, which is operated by the Association of Universities for Research in Astronomy, Inc., for NASA, 
under contract NAS 5-26555. This research has made use of the NASA/IPAC Infrared Science Archive, which is operated by the Jet Propulsion Laboratory, California Institute of Technology, under contract with NASA. This publication makes use of data from the Two Micron All Sky Survey, which is a joint project of the University of Massachusetts and the Infrared Processing and Analysis Center, funded by NASA and the NSF.
Ackerman, A. S. \& Marley, M. S. 2001, ApJ, 556, 872

Allard, F., Hauschildt, P. H., Alexander, D. R., Tamanai, A., \& Schweitzer, A. 2001, ApJ, 556, 357

Bailer-Jones, C. A. L., \& Lamm, M. 2003, MNRAS, 339, 477

Bailer-Jones, C. A. L., \& Mundt, R. 1999, A\&A, 348, 800 2001, A\&A, 367, 218

Basri, G., Mohanty, S., Allard, F., Hauschildt, P. H., Delfosse, X., Martín, E. L., Forveille, T., \& Goldman, B. 2000, ApJ, 538, 363

Burgasser, A. J., et al. 2002a, ApJ, 564, 421

Burgasser, A. J., Liebert, J., Kirkpatrick, J. D., \& Gizis, J. E. 2002b, AJ, 123,2744

Burgasser, A. J., Marley, M. S., Ackerman, A. S., Saumon, D., Lodders, K., Dahn, C. C., Harris, H. C., \& Kirkpatrick, J. D. 2002c, ApJ, 571, L151

Burgasser, A. J., et al. 2000, AJ, 120, 1100

Burrows, A., \& Sharp, C. M. 1999, ApJ, 512, 843

Clarke, F. J., Oppenheimer, B. R., \& Tinney, C. G. 2002a, MNRAS, 335, 1158

Clarke, F. J., Tinney, C. G., \& Covey, K. R. 2002b, MNRAS, 332, 361

Cooper, C. S., Sudarsky, D., Milsom, J. A., Lunine, J. I., \& Burrows, A. 2003, ApJ, 586, 1320

Delfosse, X., et al. 1997, A\&A, 327, L25

Epchtein, N., et al. 1997, Messenger, 87, 27

Geballe, T. R., et al. 2002, ApJ, 564, 466

\section{REFERENCES}

Gelino, C. R., Marley, M. S., Holtzman, J. A., Ackerman, A. S., \& Lodders, K. 2002, ApJ, 577, 433

Gizis, J. E., Monet, D. G., Reid, I. N., Kirkpatrick, J. D., Liebert, J., \& Williams, R. 2000, AJ, 120, 1085

Kirkpatrick, J. D., et al. 1999, ApJ, 519, 802

2000, AJ, 120, 447

Lunine, J. I., Hubbard, W. B., Burrows, A., Wang, Y.-P., \& Garlow, K. 1989, ApJ, 338, 314

Leggett, S. K., Allard, F., Geballe, T. R., Hauschildt, P. H., \& Schweitzer, A. 2001, ApJ, 548, 908

Leggett, S. K. et al. 2002, ApJ, 564, 452

Leggett, S. K., Toomey, D. W., Geballe, T. R., \& Brown, R. H. 1999, ApJ, 517, L139

Martín, E. L., Basri, G., Delfosse, X., \& Forveille, T. 1997, A\&A, 327, L29

Martín, E. L., Delfosse, X., Basri, G., Goldman, B., Forveille, T., \& Zapatero Osorio, M. R. 1999, AJ, 118, 2466

Martín, E. L., Zapatero Osorio, M. R., \& Lehto, H. J. 2001, ApJ, 557, 822

Nakajima, T., et al. 2000, PASJ, 52, 87

Skrutskie, M. F., et al. 1997, in The Impact of Large-Scale Near-IR Sky Surveys, ed. F. Garzon et al. (Dordrecht: Kluwer), 25

Tinney, C. G., \& Tolley, A. J. 1999, MNRAS, 304, 119

Woodward, R. C. 1992, Ph.D. thesis, Univ. Wisconsin-Madison

York, D. G., et al. 2000, AJ, 120, 1579 